\title{
Construction of Aging Model and Compensation Based on Nonlinear Time Series Analysis
}

\author{
Yi Zhang ${ }^{1,{ }^{\text {,* }} \text {, Zhi-Juan Zhang }}{ }^{1, \mathrm{~b}}$ \\ ${ }^{1}$ School of Mechano-Electronic Engineering, Xidian University, Xian 710071, China \\ a957973830@qq.com, b1692446754@qq.com
}

Keywords: quartz crystal oscillator, aging, compensation

\begin{abstract}
To solve the frequency aging problem of high-precision quartz crystal oscillator has been one of the most written topics in the international frequency control field. Aging prediction is a significant approach to the process of the long-term aging indicator problem. Aiming at the aging issue of the crystal oscillator, in this paper, we take the SC cut $10 \mathrm{MHz}$ OCXO as the aging experimental subject, construct a model by time series analysis method, fit the aging process of the crystal oscillator, predict the development trend, and compensate the accuracy of the crystal oscillator's frequency output with predicted result. The experimental results demonstrate that this model is well-performed in describing the frequency aging process of the quartz oscillator and predicting the frequency aging. Furthermore, verified by simulation, the effect of compensation is evident. The indicator, daily aging rate of crystal oscillator after compensation could reach the level of 10-11.
\end{abstract}

\section{Introduction}

In specific high-precision instruments, the frequency stability is considered an important indicator of overall performance of the system ${ }^{[1]}$. Because of the relatively stable natural frequency within quartz crystal, oscillators made of it with high precision is widely used in the instruments mentioned above. However, there is a phenomenon of frequency aging in quartz crystal oscillator, which is, the physical phenomenon of the crystal's resonant frequency increasing or decreasing monotonically with working time ${ }^{[2]}$. When the crystal is better temperature compensated ${ }^{[3-6]}$, the impact of aging drift will become the most important factor of the long-term frequency stability ${ }^{[7]}$ of crystal oscillator as the working hour extended. To solve the frequency aging problem of high-precision quartz crystal oscillator has been one of the most written topics in the international frequency control field. Aging prediction is a significant approach to the process of the long-term aging indicator problem. At present, different aging prediction models of crystal oscillator are purposed various literatures, which include logarithmic aging model ${ }^{[8]}$ (it reflects the overall tendency of the aging process moderately with terrible curve fitting), quadratic polynomial function aging model $^{[9]}$ (it only reflects the short-term aging behavior of crystal oscillator with initial data, and stop changing monotonically over time), and some other composite function models consist of elementary functions $^{[10]}$ (they are hard to realize because of the complexity within). Aiming at the 
frequency aging drift problem of the crystal oscillator, in this paper we take the SC cut $10 \mathrm{MHz}$ OCXO as the experimental subject. Based on the aging data obtained by actual measurement, we construct a mathematical model with the time series analysis method ${ }^{[11]}$ to observe the fitting degree and predict the development trend of the aging process, then calibrate the frequency output accuracy of crystal oscillator based on aging prediction results. The feasibility of this method to compensate crystal oscillator frequency drift is discussed in different existing prediction schemes. The experimental results show that the method is feasible, and the daily aging rate of SC cut OCXO after compensation could reach the level of $10^{-11}$.

\section{The Construction of Aging Models}

There are several factors result in the aging of the crystal oscillator ${ }^{[12]}$. They affect the frequency drift in different periods. This peculiarity of crystal oscillator causes the diversity in the way the crystal oscillator shows its aging characteristics ${ }^{[13,14]}$ in different phases. Therefore, try to find the aging drift rule of crystal oscillator straight from its representation is rather complicated. We regard the frequency drift ratio as a function of time, analyze the actual measurement aging data by time series analysis method, find the mathematical expression which reflects the aging process by modeling and describe the frequency aging drift tendency of the crystal oscillator in a kind of mathematic way.

Time series analysis method is a way to analyze time series based on the utilization of the past data, analogize or extend according to the development process, direction and tendency reflected by the time series, to predict the development trend of the experimental subject in the next period ${ }^{[15]}$. In practical problems, many time series models are non-linear. Various analyzations and applications of different non-linear time series models have become a research frontier and most written topics in time series analyzation. Quartz crystal oscillator's aging is a physical phenomenon, which behaves as its resonance frequency slow-varying monotonically (increase or decrease) over working time, the amplitude of frequency change rate becomes flat at the same time as the time extended. The long term changing characteristic of the curve behaves as a non-linear tendency. In the research of existing literature, some scholars proposed the linear prediction model: such as $y=k \cdot x$, this model is simple and easy to set up, but it simplify the complex aging process of crystal to plain linear variation, which makes the error becomes greater; for more examples, the time logarithmic model $y=a+b \cdot \ln (x)$ and quadratic polynomial model $y=a \cdot x+b \cdot x^{2}$, in practical applications, if the model fitting data is not highly related to actual measurement data, the predicted error will be rather big. Such prediction compensation data overlaying on the actual frequency of the crystal oscillator will worsen the crystal oscillator's long-term stability indicator of frequency output accuracy. Therefore, based on the past model research, in this paper, we propose the exponential autoregressive time series model as our frequency aging drift model of quartz crystal oscillator after several experiments.

The general exponential autoregressive time series model is as shown below:

$$
x_{t}=\sum_{j=1}^{p} f_{j}\left(x_{t-1}\right) \cdot x_{t-j}+\varepsilon_{t}
$$

$f_{j}\left(x_{t-1}\right)$ is the non-linear function of variable $x_{t-1} \cdot \varepsilon_{t}$ is an independent identically distributed white Gauss noise and $E\left(\varepsilon_{t}\right)=0, E\left(\varepsilon_{t}^{2}\right)=\sigma^{2}$. We can obtain different exponential autoregressive model by substituting $f_{j}\left(x_{t-1}\right)$ into different non-linear functions. Set $f_{j}\left(x_{t-1}\right)=a_{j}+b_{j} \cdot \exp \left(-r \cdot x_{t-1}{ }^{2}\right), j=1,2, .$. , we can obtain the exponential autoregressive model. In this paper, SC cut $10 \mathrm{MHz}$ OCXO WWH065 is chosen as the aging experimental subject. Its actual 
measurement frequency aging drift data in 182 days serves as actual measurement data of aging characteristic curve of the crystal oscillator. We fit the actual data with different models. The outcome is shown in Fig.1, with power-up working time of the crystal oscillator on the $x$ label and frequency change rate, which is $\frac{f-f_{0}}{f_{0}}$ on the $y$ label:

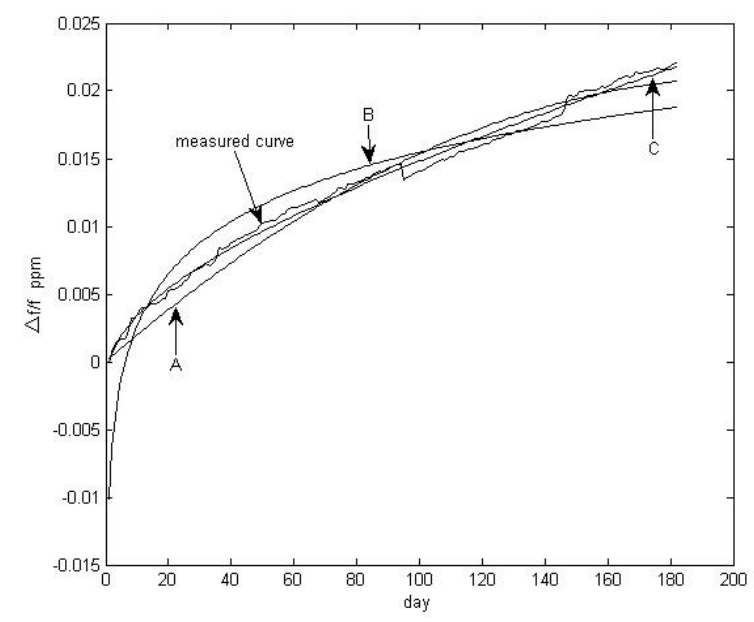

Fig.1 model fitting curve

In this figure, curve A represents the quadratic polynomial function model fit curve, curve B represents the time logarithmic function model fitting curve, curve $\mathrm{C}$ represents the exponential autoregressive time series model fitting curve proposed in this paper. As shown in the figure, curve $\mathrm{C}$ is most similar to the actual measurement data, the degree of fitting is superior to the two other models. Furthermore, we can obtain the fit relevancy of curve A, B, and C by fitting relevancy computational formula $\rho=\frac{\sum_{i=1}^{N}\left(\mathrm{x}_{i}-\overline{\mathrm{x}}\right)\left(\mathrm{y}_{i}-\overline{\mathrm{y}}\right)}{\sqrt{\sum_{i=1}^{N}\left(\mathrm{x}_{i}-\overline{\mathrm{x}}\right)^{2}\left(\mathrm{y}_{i}-\overline{\mathrm{y}}\right)^{2}}}$, which is $0.9887,0.9414$ and 0.9979 respectively. The data shows the exponential autoregressive time series model indeed fit the aging tendency curve of crystal oscillator better. Not only the fitting quality of the models in the existing literature is taken into consideration, but also the advantage in aging prediction.

\section{Aging Prediction}

In this paper, we take the actual measurement frequency aging drift data of out experimental subject, crystal oscillator WWH065 in 200 days as the actual measurement data to obtain the aging characteristic curve of this crystal oscillator. We fit the data respectively with the quadratic polynomial function model $y=a \cdot x+b \cdot x^{2}$, the logarithmic function model $y=a+b \cdot \ln (x)$, and the exponential autoregressive time series model, then predict the aging tendency in the upcoming 200 days. The fitting result is shown below in Fig.2, with the crystal oscillator power-up working time on the $x$ label and frequency change rate on the $y$ label: 


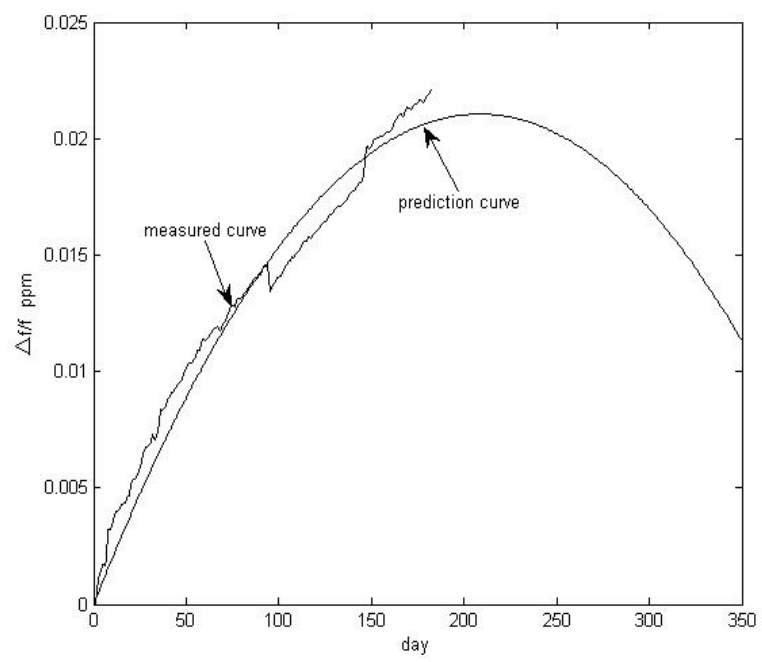

(a) quadratic polynomial function fitting prediction curve

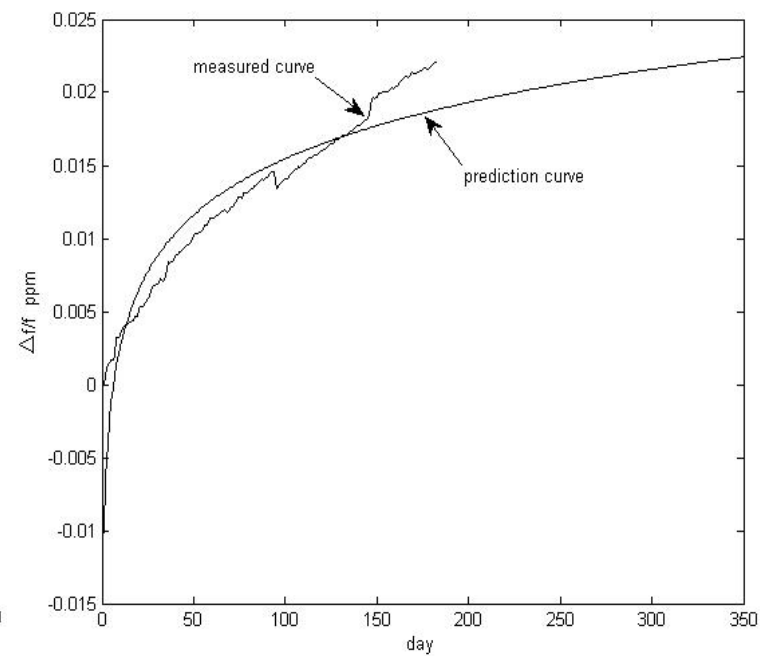

(b)logarithmic function fitting prediction curve

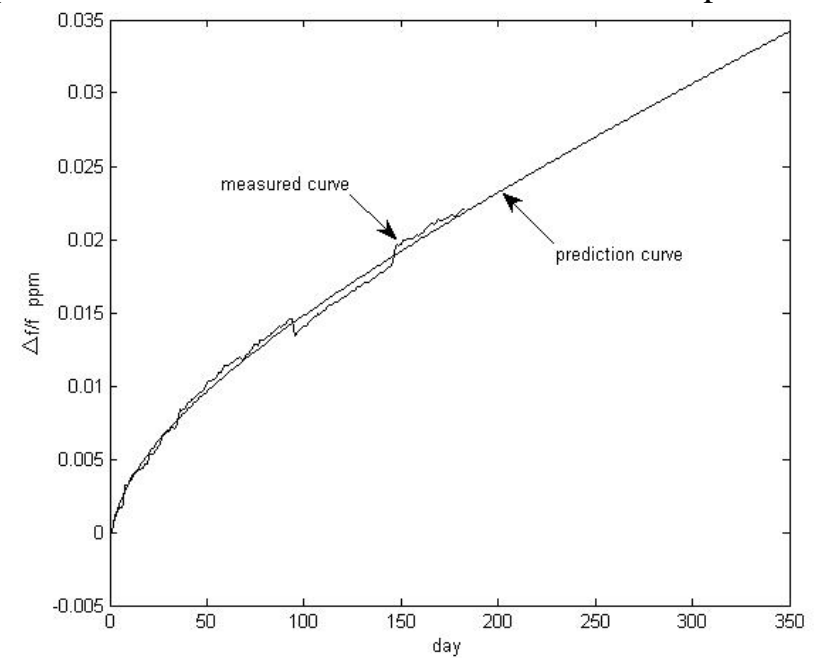

(c)exponential autoregressive function fitting prediction curve

Fig.2 Model fitting prediction curves

As shown in Fig.2, with the increase of working time, the crystal frequency presents a tendency which changes monotonically ${ }^{[17]}$. Compare the three model fitting prediction curves, we can draw a conclusion that the exponential autoregressive time series model is not only the closest to the actual data, which means it has an advantage in curve fitting quality but also the best in aging prediction, most suitable in reflecting the real frequency aging drift tendency of quartz crystal oscillator. Therefore, the last model is rather competent in describing the rule of crystal frequency aging.

\section{Compensation Model Simulation}

The main idea of prediction analysis method in solving the frequency aging of quartz crystal oscillator is: Based on the actual past data of oscillator frequency, predict the current frequency and future frequency, extract the essential compensation frequency by correlation computation with prediction aging rate data, output after overlying with the actual frequency of the oscillator. In the crystal oscillator with high stability, the frequency drift is improved over the power-up time. This fact that average frequency changes monotonically because of the aging phenomenon of frequency source's internal instrument has a characteristic similar to a system error. The frequency drift error can be obtained by time series prediction analyzation, with it the accuracy of frequency source can be corrected or calibrated. 


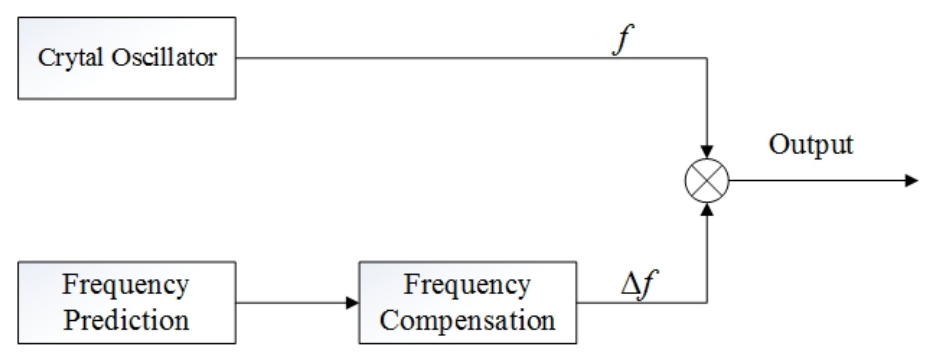

Fig. 3 prediction compensation

In this paper, the frequency drift prediction curve of the crystal oscillator is obtained by the exponential autoregressive time series model. The compensation frequency is computed from the frequency drift data which extracted from the curve, before overlying to the output of the crystal oscillator. In order to compare the prediction aging drift data with actual measurement data more conveniently, we predict the upcoming frequency drift data by the data in the first 90 days of the crystal oscillator. After a series of computations, the result which presents the aging drift curve and the aging compensation curve of the constant temperature crystal oscillator is shown in Fig.4:

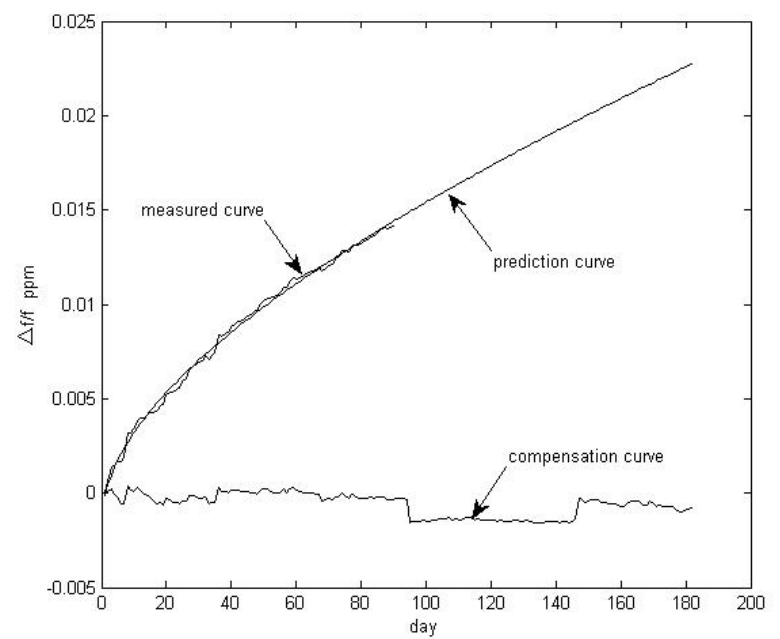

Fig.4 Aging drift curve and aging compensation curve of the crystal oscillator

In Fig.4, not only the crystal oscillator's change tendency of prediction aging drift and actual aging drift are manifested, so is the compensation result which obtained from the aging drift prediction data of mathematical model construction. We can find during the process of the computation that before the compensation, the maximum frequency aging drift rate is $0.02209 \mathrm{ppm}$, that becomes $0.001597 \mathrm{ppm}$ after the compensation. The index, diurnal aging rate of crystal oscillator after compensation could reach the level of $10^{-11}$. The result proves the exponential autoregressive time series aging model in this paper lower the error between frequency aging drift value and actual value generally, describe the frequency aging process of the crystal oscillator quite well. The prediction model could reasonably reflect the overall aging drift tendency of the constant temperature crystal oscillator, and make an effective prediction of the frequency aging.

\section{Conclusion}

Aiming at the frequency aging drift problem of the quartz crystal oscillator, we use the nonlinear time-varying exponential autoregressive prediction model in time series analysis method and compare it with some other models. The result shows that linear model is not suitable for the long-term change of the indicator of crystal oscillator, because the frequency drift value of the crystal oscillator presents a non-linear tendency with the extension of power-up working time; the quadratic polynomial function model fits the initial data, and could be used for short-term frequency 
aging drift prediction. However, its range of application is bounded and will increase the error over time. The logarithmic model is applicable in the prediction model. Although it could reflect the overall tendency of the aging curve, the error between its fitting value and the actual value is quite significant. The exponential autoregressive time series aging model in this paper is applicable to various types of the crystal oscillator aging model with small errors. This model can describe the frequency aging process of the crystal oscillator quite well and prediction the frequency aging effectively. Verified by simulation, the result of the compensation is evident. The diurnal aging rate of crystal oscillator after compensation could reach the level of $10^{-11}$.

\section{References}

[1] M Brahmananda Reddy, S. Swarna, Priskala, M. Chandrashekar, C. Vinod, P. M. Dhruva, D. K Singh. High Frequency OCXO for Space Applications[C]. 2012 IEEE International Frequency Control Symposium Proceedings, 19 July 2012, 1-5.

[2] Xiao-san Yin, Chun-nan Shen. The Study of Aging Frequency Drift Mechanism for Quartz Crystal Resonators[C]. 2013 Symposium on Piezoelectricity, Acoustic Waves, and Device Applications, Oct. 25-17, 1-4.

[3] Matko, V., Jezernik, K. New Quartz Oscillator Switching Method for Nano-Henry Range Inductance Measurements[J]. Sensors 2012, 12, 3105-3117.

[4] Matko, V., Milanović, M. High Resolution Switching Mode Inductance-to-Frequency Converter with T emperature Compensation[J]. Sensors 2014, 14, 19242-19259.

[5] Wan-thai Hsu. Reliability of Silicon Resonator Oscillators[C]. 2006 IEEE International Frequency Control Symposium and Exposition, 08 January 2007, 389-392.

[6] Matko, V. Next Generation AT-Cut Quartz Crystal Sensing Devices[J]. Sensors 2011, 11, 4474-4482.

[7] A. G Nikonov, Y. A. Ivanov. System for Evaluation of Long-term Stability of Precision Quartz Crysta l Oscillators and Results of its use[C]. 2012 European Frequency and Time Forum, 13 June 2013, 275-27 8.

[8] Shao-yun WANG, Bernd NEUBIG, Katsuyuki SATO, Tomoyuki HO SODA, Eberhard SEYDEL, Jing-hui WU, Ting-feng MA. Aging Model and Parameters of Quartz Crystal Resonators and Oscillators[C]. 2015 Symposium on Piezoelectricity, Acoustic Waves, and Device Applications (SPAWDA), 28 December 2015, 382-385.

[9] J. K. Hwang, D. H. Shin, W. Han, Y. K. Kim. A Frequency Model of OCXO for Holdover Mode of DP-PLL[C]. SICE 2000, 26-28 July 2000, 35-40.

[10] Eberhard Seydel. Relation Between Physical Processes and Aging[C]. Frequency Control Symposium 2009 Joint with the 22nd European Frequency and Time forum. IEEE International, pp. 927-930, 2009, ISSN 1075-6787.

[11] Rigoberto Fonseca, Pilar Gomez. Automatic Model Selection in Ensembles for Time Series Forecasting[J]. IEEE Latin America Transactions, vol. 14, no. 8, Aug. 2016, 3811-3819

[12] Shao-yun Wang, Bernd Neubig, Jing-hui Wu, Ting-feng Ma, Jian-ke Du, Ji Wang. Extension of the Frequency Aging Model of Crystal Resonators and Oscillators by the Arrhenius Factor[C]. 2016 Symposium on Piezoelectricity, Acoustic waves, and Device Applications, 26 January 2017, 269-272.

[13] Chun-nan SHEN, Xiao-wei YANG, Colin CHANG, Min-chiang CHAO. The Study of Activation Energy(EA) by Aging and Hign Temperature Storage for Quartz Resonator's Life Evaluation[J]. Proceedings of the 2010 Symposium on Piezoelectricity, Acoustic Waves and Device Applications, 07 April 2011, 118-122.

[14] Martin Bloch, John Ho, Oleandro Mancini, Louis Terracciano, and Leo A. Mallette. Long-Term Frequency Aging for Unpowered Space-Class Oscillators[J]. IEEE Transactions on Ultrasonics, Ferroelectrics, and Frequency Control, vol. 56, no. 10, October 2009, 2073-2078.

[15] Seng Hansun. A Comparative Study on WEMA and H-WEMA Forecasting Methods in Time Series Analysis[C]. 2016 6th International Annual Engineering Seminar (InAES), 19 January 2017, 6-10.

[16] Tugce Balli, Ramaswamy Palaniappan. EEG Time Series Analysis with Exponential Autoregressive Modelling[C]. 2008 Canadian Conference on Electrical and Computer Engineering, 15 July 2008, 000485-000488.

[17] Lozan Spassov, Viktoria Gadjanova, Radka Velcheva, Bernard Dulmet. Short- and Long-Term Stability of Resonant Quartz Temperature Sensors[J]. IEEE Transactions on Ultrasonics, Ferroelectrics, and Frequency Control, vol. 55, no. 7, July 2008, 1626-1631. 